\title{
VORWORT
}

\section{Zur ersten Auflage}

Bei dem Abfassen des vorliegenden Grundrisses der geographischen Ortsbestimmung habe ich mir die scharf begrenzte Aufgabe gestellt, ein Lehrbuch zu liefern, das wohl in erster Linie auf die praktische Verwendung seines Inhalts Rücksicht nimmt, den Benutzer jedoch nirgends, wo ihm ein tieferes Eindringen in die Sätze und Regeln der sphärischen Astronomie erwünscht erscheint, im Stiche läßt. Gegenüber allen ähnlichen Werken desselben Inhalts finden sich daher in dem vorliegenden Bändchen fast sämtliche verwendeten Formeln und mathematischen Ausdrücke elementar abgeleitet vor. Durch eine vorausgeschickte übersichtliche $\mathrm{Zu}-$ sammenstellung der in der Ortsbestimmung gebräuchlichen trigonometrischen Sätze ist es trotzdem möglich geworden, das mathematische Gerüst des Buches auf ein Minimum zu reduzieren, das auch diejenigen, die ihr Interesse ausschließlich der praktischen Ortsbestimmung zuwenden - ich denke hier insbesondere an Geographen und Forschungsreisende - nicht abschrecken wird. Manche Vereinfachung ist dadurch erzielt worden, da $B$ nur kleine Instrumente, die günstigstenfalls etwa Winkel von 0. I Bogenminuten abzulesen gestatten, in den Kreis der Betrachtungen gezogen worden sind. Aus diesem Grunde dürfte der engbegrenzte und doch ziemlich vollständige Inhalt des Buches auch allen denjenigen, die sich später in die Aufgaben der Ortsbestimmung erheblich weiter vertiefen wollen, als erster Handweiser $e^{i n e}$ gute Grundlage bieten.

Auf die Einfügung übersichtlicher und lehrreicher Figuren ist ein besonderes Augenmerk gerichtet worden. Alle Darstellungen der Himmelskugel sind z. B. streng in orthographischer Projektion ausgeführt und zum Zwecke des besseren Verständnisses reichlich beschriftet worden, wie ich überhaupt bestrebt gewesen bin, der geometriechen Anschauung des Lesers nach Möglichkeit entgegenzukommen. 
Bei der Lesung der Korrekturen des Manuskriptes und des Satzes hat mich Herr Eisenbahnobersekretär W. Voß in Altona sehr wesentlich unterstützt. Ihm verdanke ich auch eine vollständige Durchsicht aller Formeln sowie die Ausführung einer Kontrollrechnung zu den im Anhang I und II mitgeteilten Beispielen und Tafeln, so daß für die Zuverlässigkeit des Zahlenmaterials eine gewisse Bürgschaft übernommen werden kann.

Sternwarte Bergedorf, Dezember I9I3.

\section{K. Graff}

\section{Zur zweiten und dritten Auflage}

Die zweite und dritte Auflage des Grundrisses der geographischen Ortsbestimmung erscheint auf Wunsch des Verlages in äußerlich veränderter Form. Aber auch inhaltlich weist das Buch eine Reihe von Abweichungen gegenüber der Erstausgabe auf. Es handelt sich zunächst um verschiedene Kürzungen, soweit sich diese ohne Beeinträchtigung der Klarheit der Darstellung ermöglichen ließen, oder soweit Beobachtungsverfahren in Frage kommen, die an kleinerem MeBgerät kaum noch angewendet werden bzw. durch die drahtlose Zeitübertragung gegenstandslos geworden sind. Eine Ergänzung des früheren Inhalts bildet der Abschnitt über die Zeitsignale und deren Empfang sowie die erweiterten zwei Kapitel über die genaue Azimutbestimmung.

Da das Buch den astronomischen, geodätischen und nautischen Anfänger ganz allgemein in die Behandlung sphärischer Aufgaben einführen soll, haben verschiedene Sonderaufgaben der Ortsbestimmung, wie z. B. die Zweihöhenmethode oder das Standlinienverfahren, unter Fortlassung alles Nebensächlichen in der zweiten und dritten Auflage ihren Platz behalten.

Auf Freiheit des Satzes von Druckfehlern ist wieder besonders geachtet worden. Für die beim Korrekturlesen geleistete Hilfe und die sorgfältige Kontrolle der Beispiele und Tafeln bin ich, wie bei der ersten Auflage, Herrn Amtmann W. VoB in Altona zu besonderem Dank verpflichtet.

Wien-Breitenfurt, Januar I944. 